\title{
Réparer la surface de la Terre
}

Les restes dans les rituels de construction au sein des villes mayas classique (250-950 apr. J.-C.)

Resurfacing the Earth: Leftovers in Rituals of Construction in Classic Maya Cities (AD 250-950)

\section{Marie-Charlotte Arnauld et Tristan Saint-Dizier}

\section{(2) OpenEdition}

\section{Journals}

Édition électronique

URL : https://journals.openedition.org/tc/7917

DOI : $10.4000 /$ tc. 7917

ISSN : 1952-420X

Éditeur

Éditions de l'EHESS

Édition imprimée

Date de publication : 31 octobre 2016

Pagination : 138-149

ISBN : 9782713225291

ISSN : 0248-6016

Référence électronique

Marie-Charlotte Arnauld et Tristan Saint-Dizier, « Réparer la surface de la Terre », Techniques \& Culture

[En ligne], 65-66 | 2016, mis en ligne le 31 octobre 2018, consulté le 29 septembre 2022. URL : http:// journals.openedition.org/tc/7917 ; DOI : https://doi.org/10.4000/tc.7917 


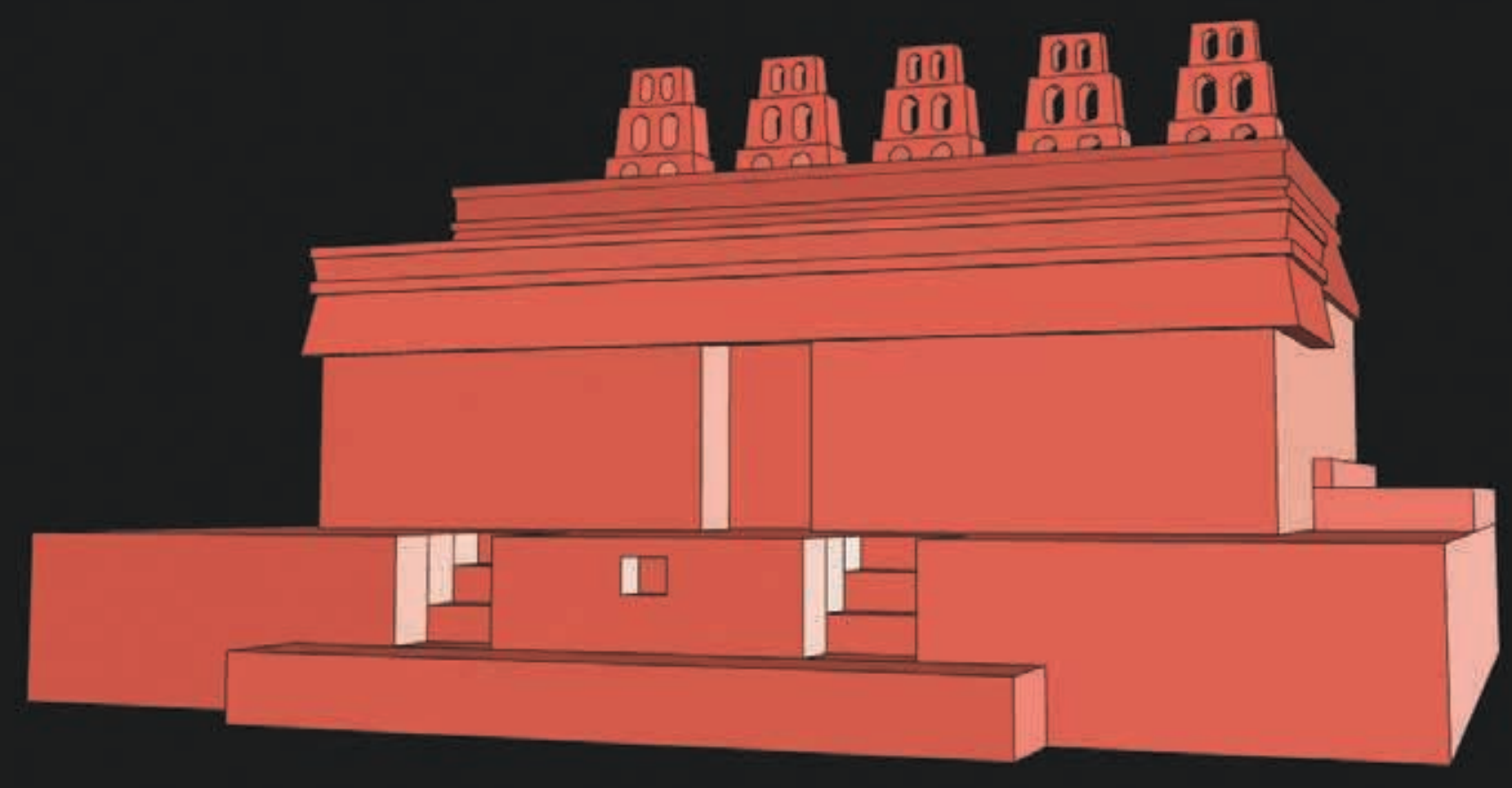




\section{Réparer la surface de la Terre}

Les restes dans les rituels de construction au sein des villes mayas classique (250-950 apr. J.-C.)

Dans certains habitats anciens, à l'extérieur ou à l'intérieur, on trouve de grandes quantités d'objets, en général brisés, éparpillés en couche, ou concentrés en fosse, dont il ne reste que les «non périssables». Restes de la vie quotidienne en rebuts et dépotoirs? Ou restes de pratiques rituelles réalisées à des moments clés? Les dépôts rituels sont fréquents en Mésoamérique (Dehouve 2007, Broda 2013) et de nombreuses sources ethnohistoriques et ethnographiques aident à détecter dans chaque cas les structures internes qui en font les témoins figés de gestes rituels liés à la construction des maisons, leur reconstruction ou leur abandon. Combiner ainsi déchets et rites reflète une conception maya de l'action humaine située dans le temps et dans l'univers qu'il convient de décrire puis d'interpréter dans sa complexité.

Voici un cas de dispositif complexe de cinq dépôts trouvés dans un bâtiment voûté du centre du site de La Joyanca (Guatemala). 
1. Les nombreux sites archéologiques (triangle noir) explorés et fouillés dans les basses terres mayas du Yucatán, et leurs habitats classiques (200-900 apr.).-C.), maisons voûtées et non voûtées

2. Restitution d'un habitat sur le site de Xculoc, Mexique
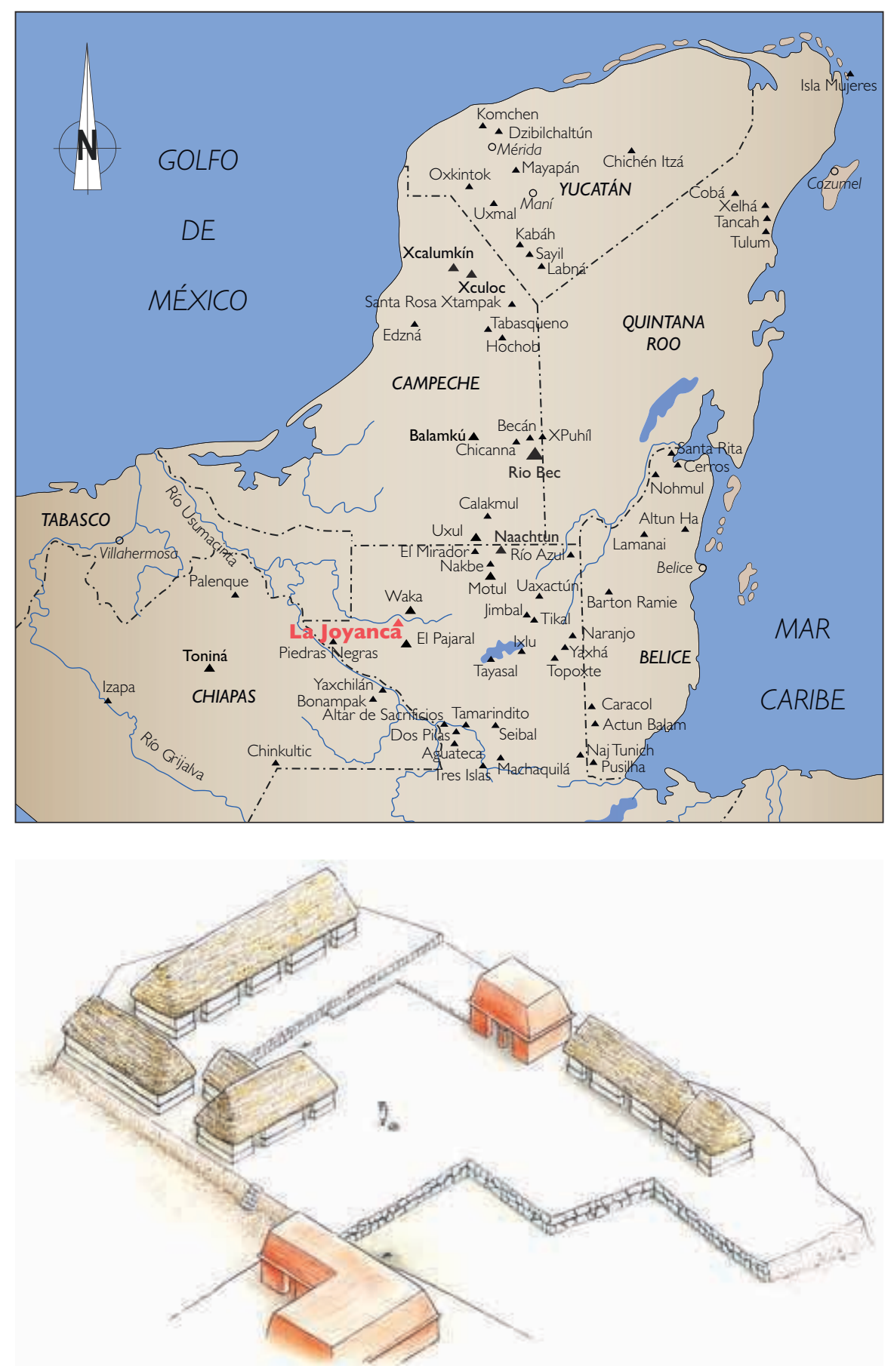
La Joyanca s'est constituée par immigration vers 500-650 apr. J.-C. sur un village antérieur occupé depuis près d'un millénaire. Seul véritable lieu public, la «place principale»a été entièrement reconstruite à partir de 600 , avec sur le côté ouest un premier édifice (codé 12sub), ni temple ni maison, peut-être le substitut symbolique de l'une des résidences élitaires (Arnauld et al. 2004: chap. 5).

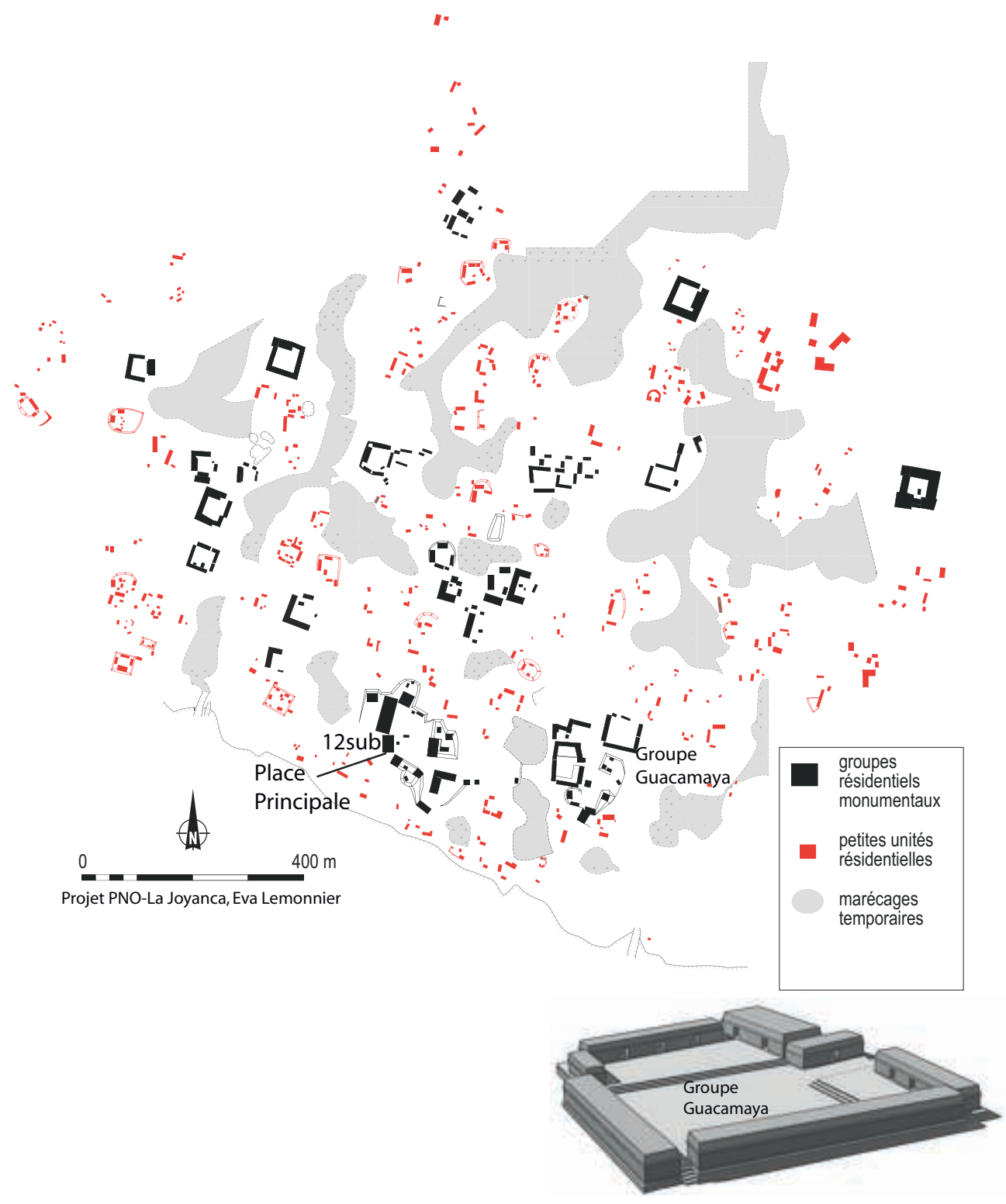

3. La ville La Joyanca vers 800 apr. J.-C., avec la place principale et une douzaine d'habitats élitaires 
La construction et l'occupation de la «place principale» (PP) visible actuellement sont datées 600-850 apr. J.-C. Trois dates radiocarbone proviennent de l'Édifice 12sub: 620, 720, 740. La céramique indique une construction en 600-650, et des éléments de l'inscription en glyphes gravée à l'intérieur situent le remblaiement final après 680. Mal construite, s'affaissant, elle n'a pas dû être occupée plus d'un siècle. Une fois remblayée, elle a été recouverte par une pyramide.

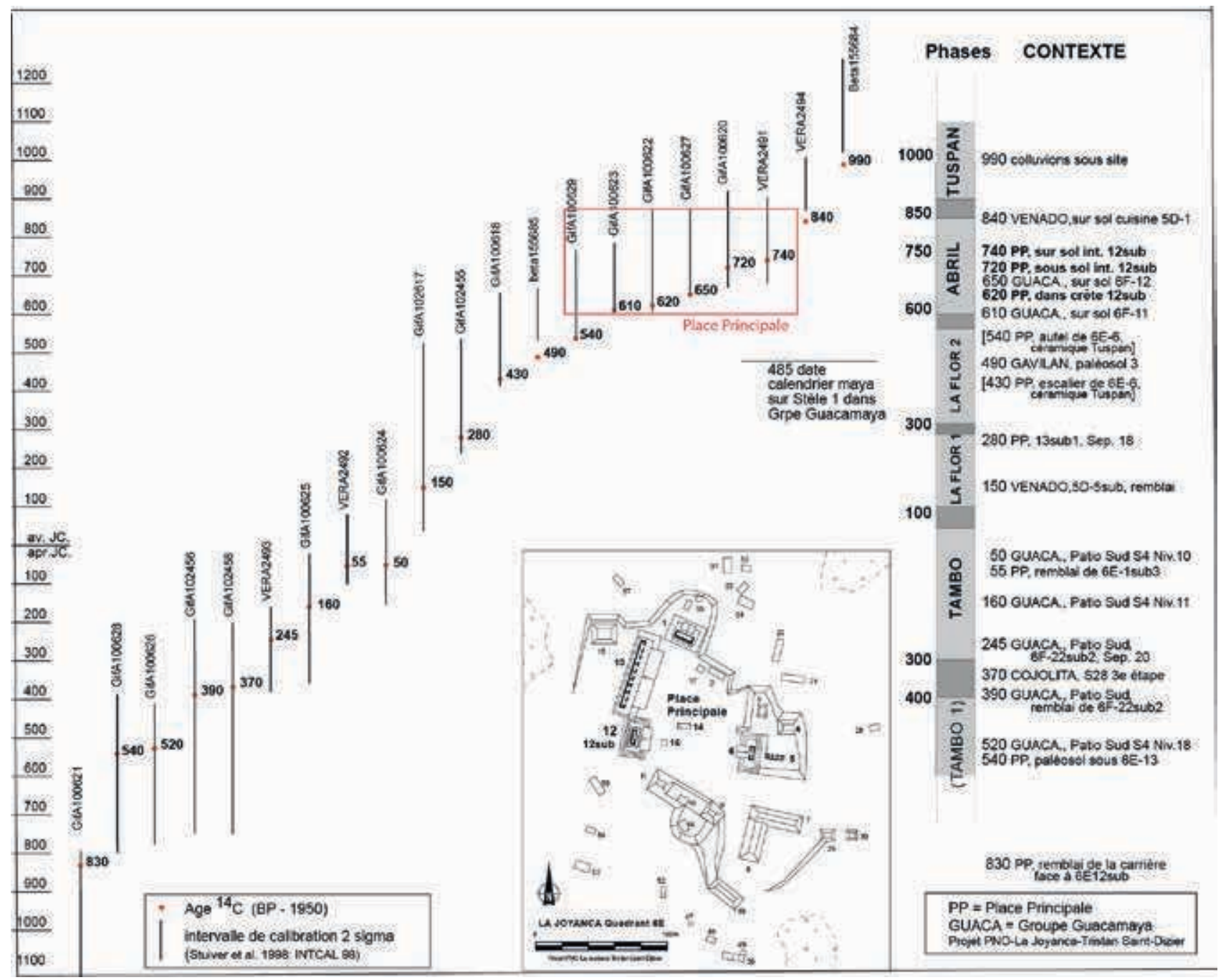

4. Chronologie radiocarbone et céramique 


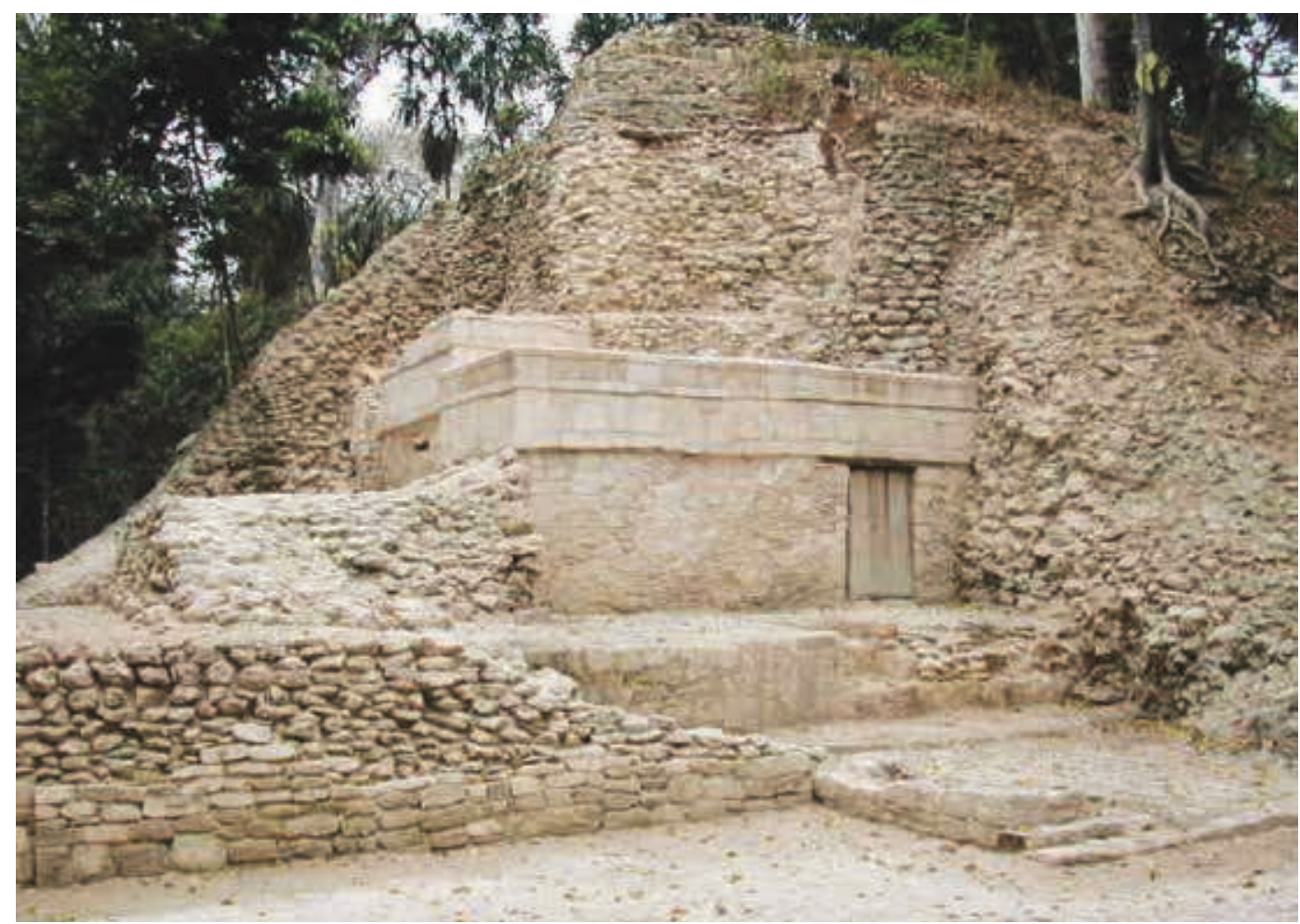

Sur le côté ouest de la place principale, l'Edifice 6E12sub a été construit sur une plateforme, dont l'escalier d'accès est situé dans l'axe de la porte unique. Il comporte une niche contenant du charbon, probable reste d'offrandes rituelles que recouvraient les vestiges de la décoration de stuc modelé de la façade. L'édifice est entièrement maçonné de pierres calcaires soigneusement taillées, et voûté de façon assez rustique. La crête faitière à éléments ajourés (voir l'image d'ouverture) est inspirée des temples de Palenque: ennoyée dans le remblai de la pyramide postérieure, elle n'était visible qu'à l'intérieur par les tranchées de pillage qui perçaient le toit. Gravement endommagée, cette petite structure n’a dû sa préservation qu’au remblai qui remplissait ses pièces.
5. L'Édifice 12sub, restitution et restauration après son difficile dégagement à l'intérieur de la Pyramide Temple 12 qui l'a recouvert vers 720-750 apr. J.-C. 

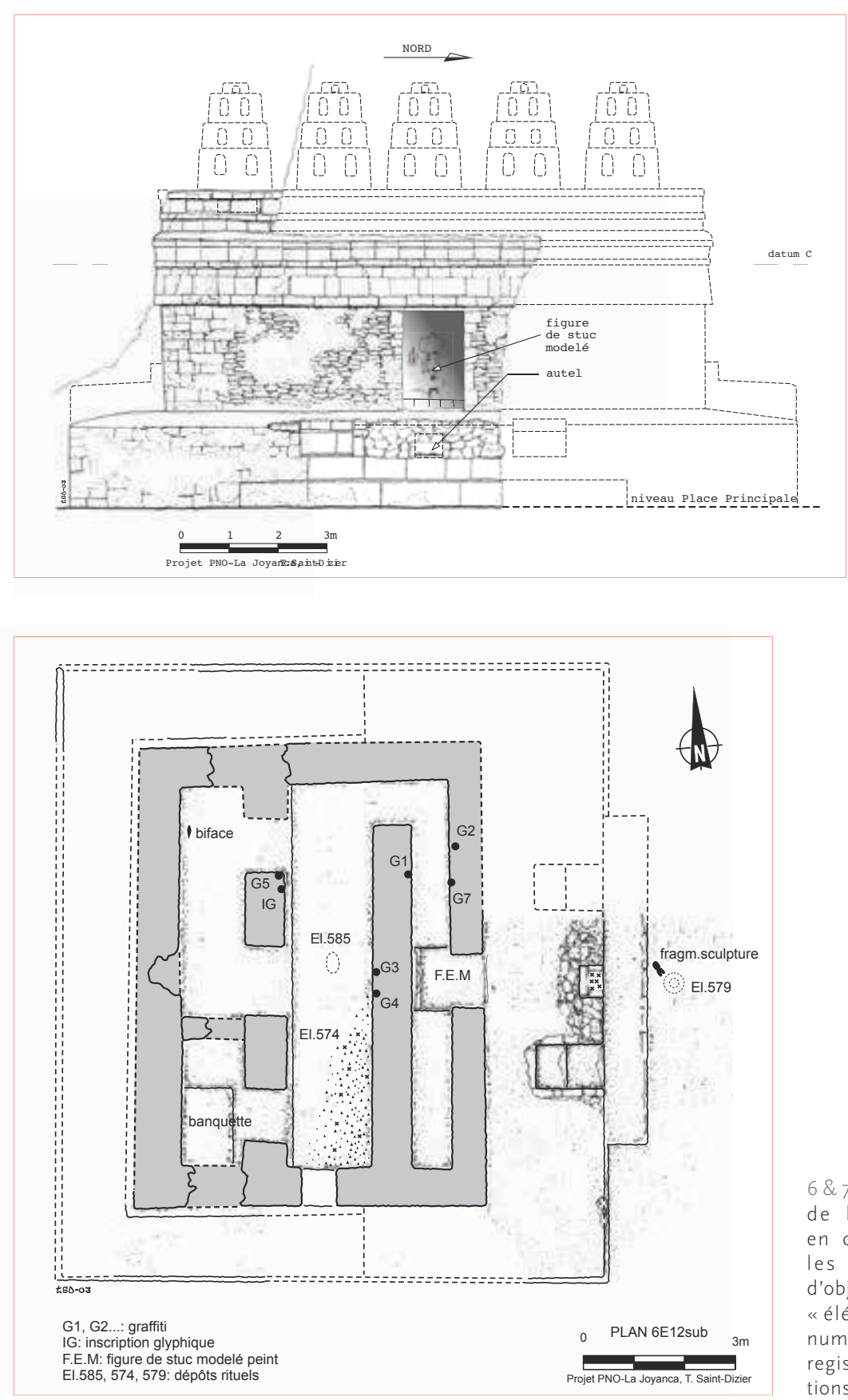

Le plan en labyrinthe, les trois pièces obscures, les graffitis à motifs religieux et la figure solaire de stuc modelé et peint face à l'entrée (FEM sur le plan) suggèrent que ce lieu réservé était une «maison sacrée» (Valdés 2001 : 142).

Nous avons découvert cinq dépôts enfouis: trois faits au moment de la construction de l'édifice, et deux juste avant son remblaiement final, sa clôture. Des premiers, dits dépôts de fondation, deux se trouvent à l'extérieur et sont associés l'un, étalé en couche, à l'autre, en fosse (Éléments 579-577); le troisième est à l'intérieur, en couche sous le sol en partie détruite par les pillages (non visible ici). Au-moment de la clôture, à l'intérieur, sont aussi associés deux dépôts, l'un en couche sur le sol (574) et l'autre en fosse (585).
$6 \& 7$. Élévation et plan de l'Édifice 12sub; en cours de fouille, les concentrations d'objets ont été codées «éléments» (EI.) et numérotées afin d'en. registrer les compositions précises. 

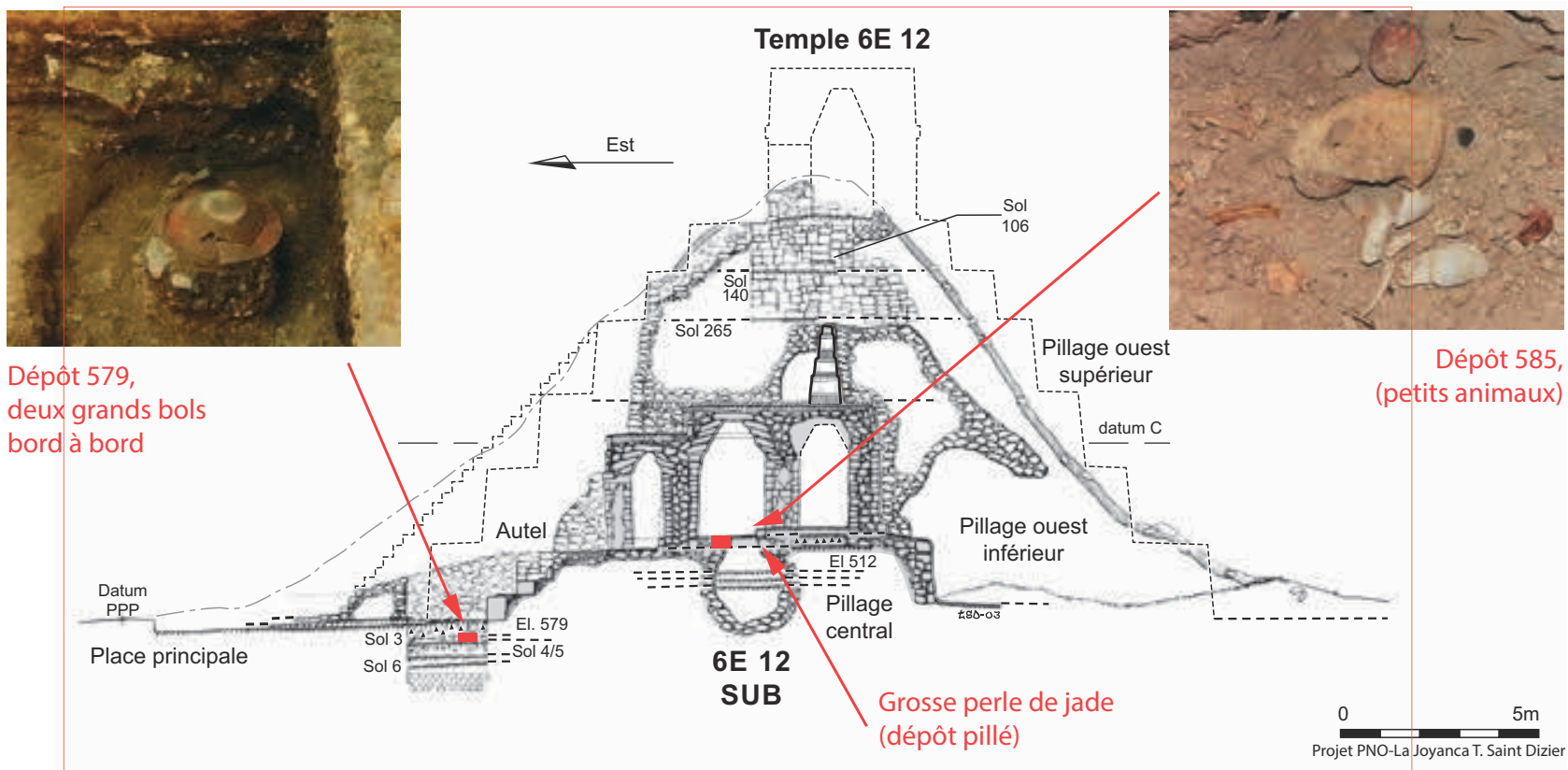

Les plus typiques des rituels mayas de fondation et de clôture (et ce, encore à ce jour) sont les dépôts concentrant les restes en fosse rebouchée. Ici:

- le dépôt de fondation extérieur (Élément 579) est dans l'axe est ouest de l'Édifice 12sub, en fosse intrusive sous le sol stuqué de la "place», dans le dépôt en couche (577); le bol inférieur sous son couvercle contenait les restes d'un très petit vertébré (Emery

8. Coupe est-ouest montrant les deux édifices superposés, extrêmement pillés; leur fouille a été difficile et dangereuse; leur restauration a duré quatre sai\& Thornton 2008).

- le dépôt de fondation intérieur en principe associé au dépôt en couche (Élément 512) aurait été pillé; il en resterait une grosse perle de jade trouvée au centre.

- le dépôt de clôture intérieur se trouvait dans une petite fosse centrale (585), près du dépôt en couche associé (574), couverte de trois pierres (sol de la pièce percé); elle contenait 33 individus entiers, très petits ou juvéniles, tous d'espèces animales, aquatiques et volatiles (eau et air), non chassées ni comestibles (Emery \& Thornton 2008) et un fragment de jade poli. 
Q. Coupe est-ouest du seul Édifice 12 sub avec les dépôts en couche.
Voici la séquence des gestes rituels:

1. Fondation, à l'extérieur: avant de construire le sol de la Place et la plate-forme de 12sub, les constructeurs ont étalé une couche (Élément 577) de terre noire avec esquilles de charbon, type «sol cultivé», avec de nombreux tessons dont un plat brisé dispersé, des éclats de silex et un fragment de molette, sans aucun ossement animal hormis ceux du dépôt associé en fosse (579).

2. Fondation, à l'intérieur: une fois la plate-forme construite mais juste avant de faire son sol intérieur stuqué, ils ont épandu la couche (512) de terre charbonneuse (2-14 cm épaisseur), type «vidange de foyer», avec de très nombreux tessons (des remontages possibles), et une lame de silex (fouille partielle; le dépôt en fosse associé, s'il a existé, a dû être pillé).

3. Clôture, à l'intérieur: juste avant le remblaiement final, en même temps que le dépôt des 33 animaux en fosse, ils ont étalé sur le sol de la pièce centrale la couche (574) de tessons mêlés à du charbon concentré (575) et à des cendres, sans ossements animaux (fouille exhaustive)

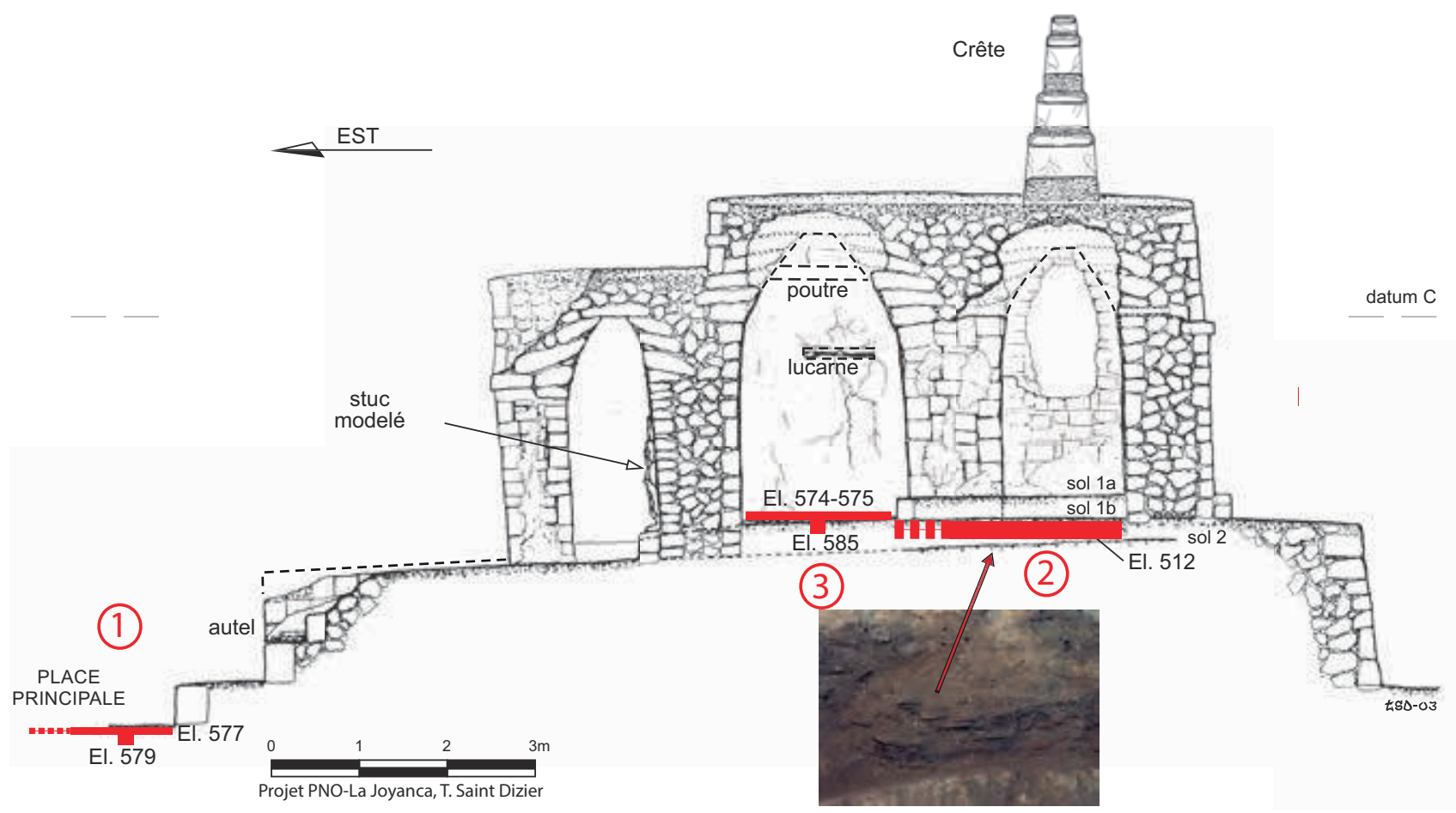



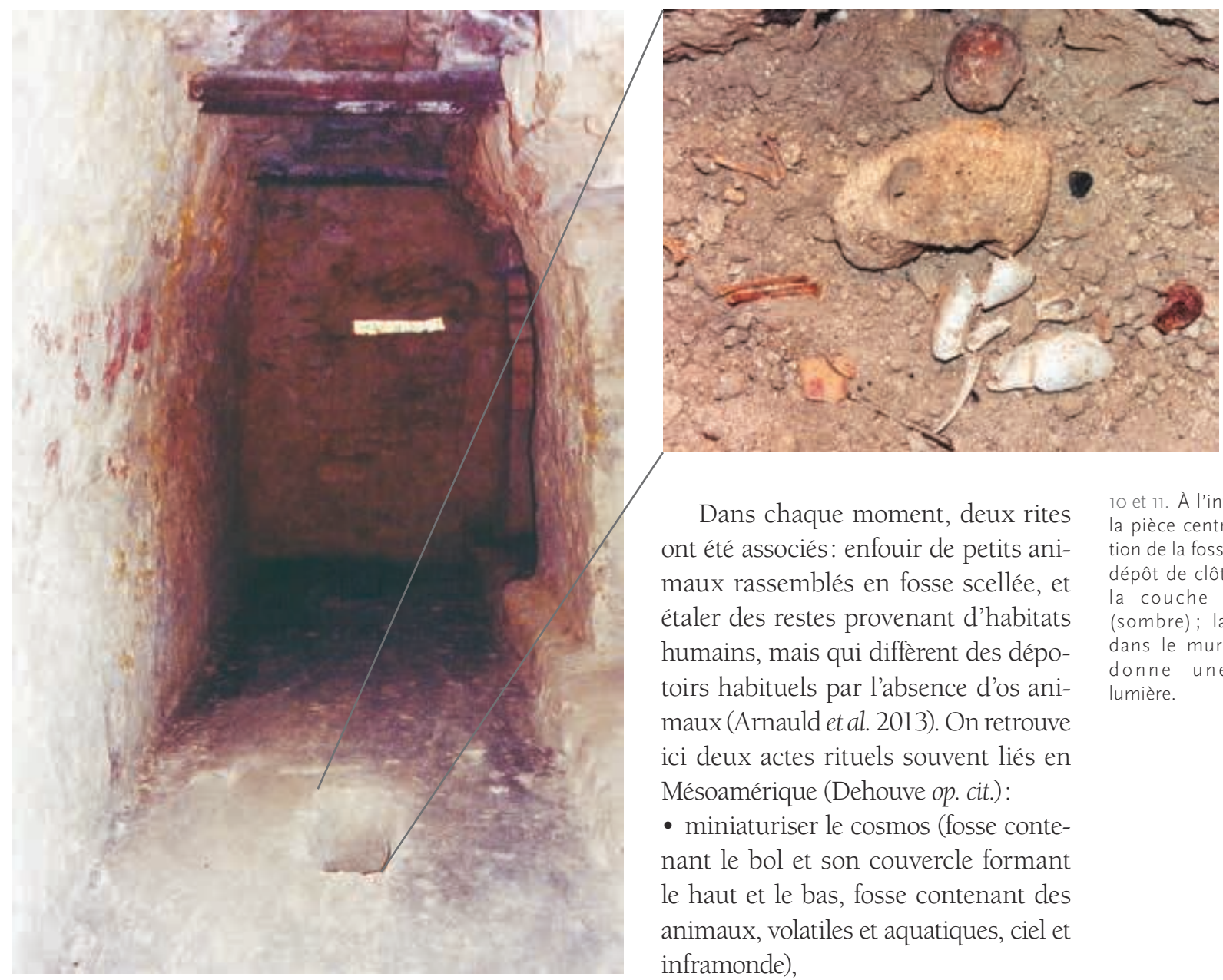

Dans chaque moment, deux rites ont été associés: enfouir de petits animaux rassemblés en fosse scellée, et étaler des restes provenant d'habitats humains, mais qui diffèrent des dépotoirs habituels par l'absence d'os animaux (Arnauld et al. 2013). On retrouve ici deux actes rituels souvent liés en Mésoamérique (Dehouve op. cit.):

- miniaturiser le cosmos (fosse contenant le bol et son couvercle formant le haut et le bas, fosse contenant des animaux, volatiles et aquatiques, ciel et inframonde),

- constituer une scène (au sens théâtral) à l'action humaine collective que représentent l'édifice par sa construction puis l'activité qu'il abrite, réparer les désordres dus à cette action à la surface de la Terre (épandage de terre de jardin, vidanges de foyers domestiques, dépotoirs céramiques et lithiques, dont on a extrait tout os animal).

Sur le lieu public, au petit Édifice 12sub ont succédé un temple sur pyramide, ainsi qu'une maison longue de gouvernement, partie des bâtiments institutionnels de la ville. La maison sacrée (12sub) en assurait l'ancrage social et cosmique par l'effet des actions rituelles de fondation et de clôture réalisées dans sa structure bâtie.

Car au-delà des enjeux locaux de La Joyanca, le sens de ces pratiques est connu par l'ethnographie maya: les offrandes enfouies dans la Terre «nourrissent » la résistance de la
10 et 11. A l'intérieur de la pièce centrale, position de la fosse (585) du dépôt de clôture et de la couche associée (sombre); la lucarne dans le mur du fond donne une faible lumière. 


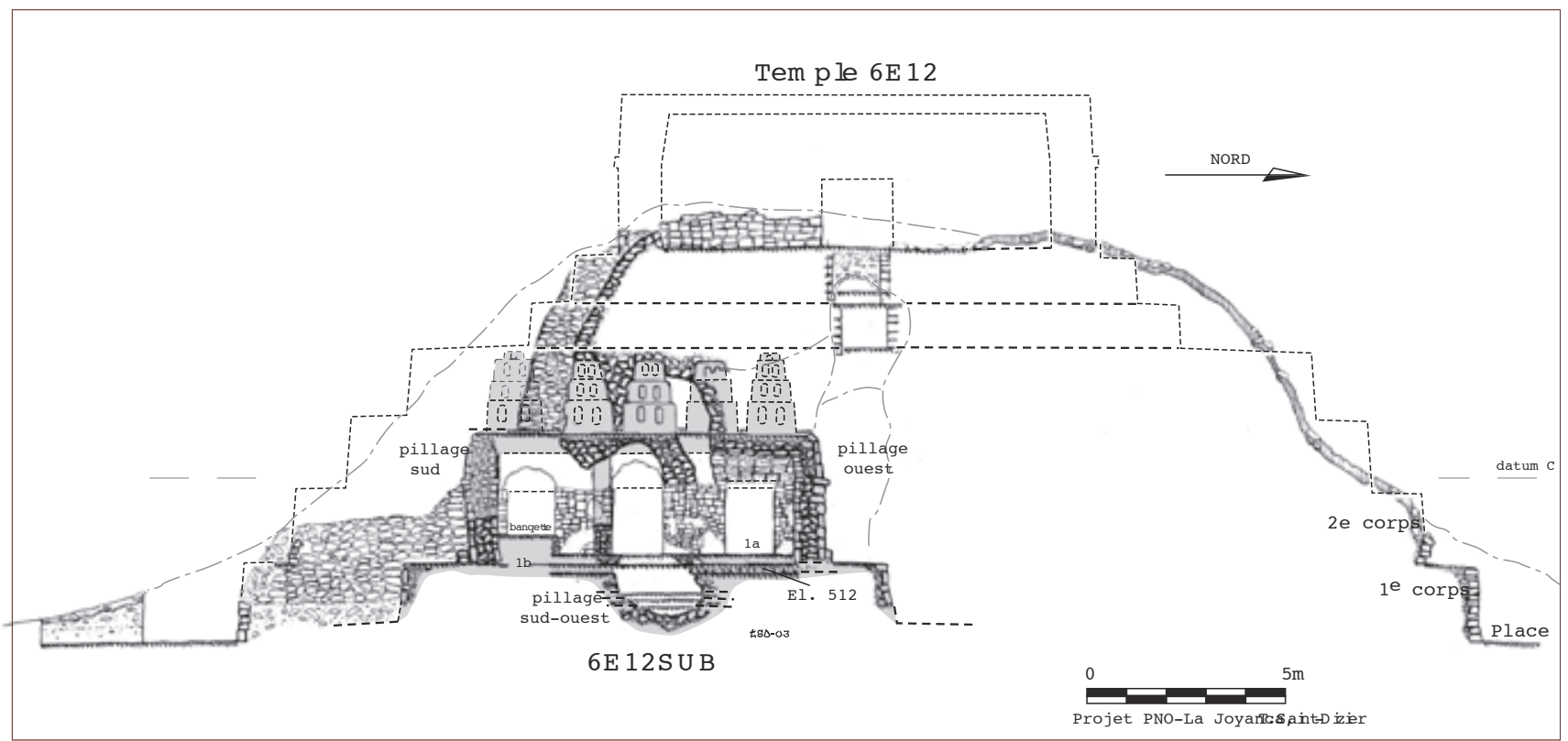

12. Coupe nord-sud de la pyramide-temple 6E12 englobant l'édifice 6E12sub.

13. Le côté ouest de la «place principale», Édifices $6 \mathrm{E}_{12}$ et $6 \mathrm{E}_{13}$

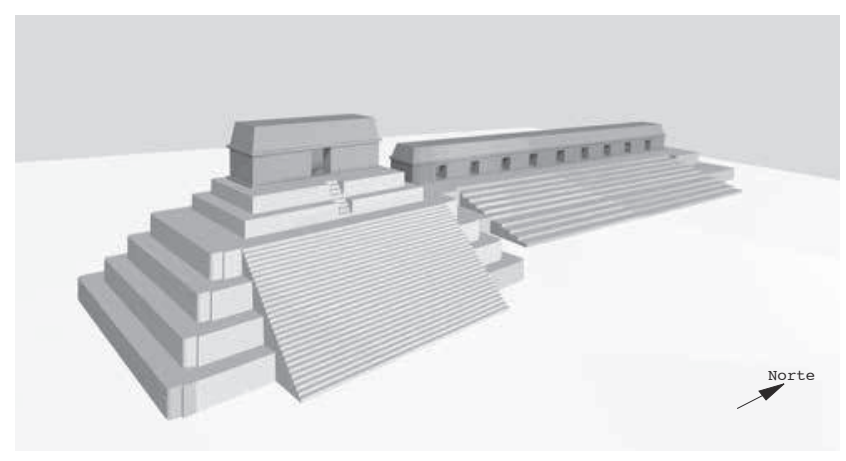

construction, tandis que les dépôts étalés sur la Terre, entre ciel et inframonde, assurent la réparation des dégâts de la construction dus à l'abattage d'arbres, aux creusements... (Brown \& Emery 2008, Carlson \& Eachus 1977, Monaghan 1996, Vogt 1965).

L'archéologie des abandons de maisons fournit de nombreux exemples de restes domestiques étalés rituellement (voir à ce sujet, Chase \& Chase 2004), peut-être dans la même intention de « cicatriser» les traces de la présence humaine. Cette pratique rituelle peut avoir aussi d'autres connotations tenant à la gestion des déchets et à l'amendement des sols en agriculture intra-urbaine maya classique.

Nous remercions Véronique Breuil-Mártinez, Eva Lemonnier (Projets PNO-La Joyanca), Kitty Emery (Florida Museum of Natural History) et Sylvie Elies (ArchAm). 


\section{Les auteurs}

Marie-Charlotte Arnauld est archéologue spécialiste de la Mésoamérique (fouilles dans l'aire maya), équipe «Archéologie des Amériques» (ArchAm, UMR8096 CNRS). Ses recherches portent actuellement sur la temporalité des rituels, la mobilité des populations et les crises environnementales en Mésoamérique, en particulier dans 7 sites archéologiques des basses terres mayas dont La Joyanca.

Tristan Saint-Dizier, architecte DPLG, participant aux campagnes de fouilles du projet PNO-La Joyanca (1999-2003), a réalisé à ce titre relevés et dessins dont ceux de la Structure 6E12sub rendue difficile par de nombreux tunnels de pillage et rédigé un mémoire sur l'aménagement du site pour le public doté de recommandations mises en place sous sa direction.

\section{Iconographie}

Image d'ouverture. l'Edifice 12sub. (C) Projets La Joyanca, CNRS, ArchAm, UMR8096.

Plans, dessins et modèles 3D. (C) T. Saint-Dizier.

\section{Références}

Arnauld, M.-Ch., Breuil-Martinez, V. \& E. Ponciano 2004 La Joyanca (La Libertad, Guatemala), antigua ciudad maya del noroeste del Petén. Mexico et Guatemala: CEMCA, CIRMA, Asociación Tikal.

Arnauld, M.-Ch., Forné, M., Ponciano, E., Lemonnier, E. et al. 2013 «Ritos de transición, ritos de crísis: perspectivas para la movilidad poblacional en las sociedades mayas clásicas» in Barbara Arroyo et al. dir. XXVI Simposio de Investigaciones Arqueológicas en Guatemala, 2012, Ciudad Guatemala: Museo Nacional de Arqueología y Etnología.

Broda, J. dir. 2013 Convocar a los dioses: ofrendas mesoamericanas. Estudios antropológicos, históricos y comparativos. Instituto Veracruzano de la Cultura, Conaculta, Xalapa.

Brown, L. \& K. Emery 2008 «Negociations with the Animate Forest: Hunting Shrines in the Guatemala Highlands», Journal of Archaeological Method and Theory 15: 300-337.

Carlson, R. \& F. Eachus 1977 «The Kekchi Spirit World» in Helen L. Neuenswander et Dean Arnold (dir.) Cognitive Studies of Southern Mesoamerica, Dallas: Summer Institute of Linguistics Museum of Anthropology3: 35-65.
2. (C) Projet Xculoc, CNRS, ArchAm, UMR8096.

Autres images. (C) Projets La Joyanca, CNRS, ArchAm, UMR8096.

Chase, D. Z. \& A.F. Chase 2004 «Hermeneutics, transitions and transformations in Classic to Postclassic Maya Society» in Arthur A. Demarest, Prudence M. Rice $\&$ Don S. Rice dir. The Terminal Classic in the Maya Lowlands: Collapse, Transition, and Transformation. Boulder: University of Colorado Press: 12-27.

Dehouve, D. 2007 Offrandes et sacrifices en Mésoamérique. Paris: Riveneuve.

Emery, K. \& E. Thornton 2008 « Reporte preliminar de los restos faunísticos recuperados en el sitio de La Joyanca», Environmental Archaeology Program Florida Museum of Natural History. Ciudad Guatemala: Instituto de Antropologia e Historia de Guatemala.

Monaghan, J. 1996 «Fiesta finance in Mesoamerica and the origins of a gift exchange system », Journal of the Royal Anthropological Institute 2: 499-516.

Valdés, J.A. 2001 «Palaces And Thrones Tied to the Destiny of the Royal Courts in the Maya Lowlands » in Takeshi Inomata \& Stephen D. Houston dir. Royal courts of the Ancient Maya. Volume II: Data and Case Studies, Boulder and Oxford: Westview Press: 139-164.

Vogt, E.Z. 1965 Tortillas for the Gods: A Symbolic Analysis of Zinacanteco Rituals. Norman: University of Oklahoma.

\section{Pour citer cet article}

Arnauld, M.-C. \& T. Saint-Dizier 2016 « Réparer la surface de la Terre. Les restes dans les rituels de construction au sein des villes mayas classique (250-950 apr.J.-C.)», TechniquesECulture 65-66 «Réparer le monde. Excès, reste et innovation», p. 138-149. 